\title{
Detection of OXA Carbapenemase Positive Acinetobacter spp., Jamaica C-A Thoms-Rodriguez ${ }^{1}$, T Mazzulli ${ }^{2,3}$, N Christian ${ }^{1}$, BM Willey $^{2}$, AM Nicholson ${ }^{1}$
}

\begin{abstract}
Objective: The global problem of resistance to carbapenem antibiotics, through the production of carbapenemases by clinically significant bacteria, continues to increase while options for antibiotic therapy remain limited. It is important to determine the mechanism of carbapenem resistance in the multidrug resistant (MDR) Acinetobacter spp. isolated at a tertiary care hospital, Jamaica (TCHJ) because of the implications for therapy which is the goal of this study.

Methodology: 82 MDR Acinetobacter spp collected at a TCHJ were identified as potential carbapenemase producers during routine susceptibility testing for a one year period from May 2009 to April 2010. These isolates were subjected to phenotypic and genotypic tests for carbapenemase detection using the modified Hodge test and PCR respectively. Multiplex PCR for OXA-23, -24, -51, -58 was performed and PFGE was used to determine if carbapenemase-positive isolates were related.

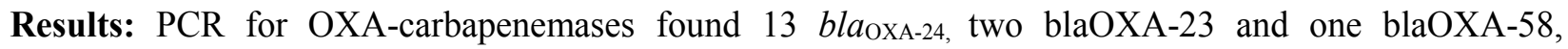
establishing a prevalence of $19.5 \%$. PFGE results showed that Acinetobacter spp sharing phenotypic and genotypic similarites were clonally related.
\end{abstract}

Conclusions: Carbapenemase production was found to be a cause of antibiotic resistance in MDR Acinetobacter spp. isolates at a TCHJ. These isolates were thought to have been derived from an outbreak or endemic strain whose presence is likely to significantly impact patient management and antibiotic policy.

Keywords: Acinetobacter spp, carbapenem, OXA

From: ${ }^{1}$ Department of Microbiology, The University of West Indies, Kingston, Jamaica, ${ }^{2}$ Department of Microbiology, Mount Sinai Hospital/University Health Network, Toronto, Ontario, Canada, ${ }^{3}$ Department of Laboratory Medicine and Pathobiology, University of Toronto, Toronto, Ontario, Canada.

Correspondence: Dr A Nicholson, The University Hospital of the West Indies/ The University of the West Indies, Kingston, Jamaica. Fax: 1-876-9779553, e-mail: alison.nicholson@uwimona.edu.jm

\section{INTRODUCTION}


The challenge faced by clinicians to find suitable therapy for multidrug resistant (MDR) pathogens causing infections is a global one (1). At a tertiary care hospital, Jamaica (TCHJ) where this study was done, Gram-negative bacilli have been implicated in $72.5 \%$ of infections in patients admitted to the intensive care unit (2). While Acinetobacter spp. isolated in the unit between 2002 and 2004 showed resistance to multiple antibiotics including the carbapenems (2), the mechanisms of resistance, crucial to antibiotic choice and appropriate infection control management were not elucidated.

Acinetobacter spp. are opportunistic pathogens that colonize human skin and dry surfaces (3). These characteristics and their ability to produce carbapenemases, allow them to resist agents such as antibiotics allowing them to establish themselves as important nosocomial pathogens (3). There have been reports in the literature of carbapenem antibiotics being used to manage Acinetobacter spp. infections. (2). However in recent times, as resistance to this class of antibiotic increases through the dissemination of carbapenem-hydrolysing enzymes, carbapenemases, their usefulness for the treatment of infections is increasingly compromised (3, 5). The carbapenemase genes are often transferred on plasmids that carry many other resistance genes, thereby rendering the organism resistant to multiple classes of antibiotics.

The first report of a carbapenem resistant Acinetobacter spp. infection was from a patient receiving care at the Royal Infirmary of Edinburgh (4). Since then carbapenem resistance in Acinetobacter spp. has emerged in various parts of the world, mostly as a result of the dissemination of class D OXA-type carbapenemases (4). The extent to which carbapenem resistance due to OXA carbapenemases is detected among the Acinetobacter spp. varies globally. Of 221 Acinetobacter isolates (resistant to imipenem) from 11 Chinese teaching hospitals collected between 1999 and 2005, 97.7\% carried bla oxa23 -like genes (6). OXA-23-like 
carbapenemases have been detected repeatedly within Acinetobacter spp. in the UK, East Asia, and South America (7). This enzyme persisted in a MDR epidemic strain that has become prevalent in UK and is known as the OXA-23 clone 1. Reports of the dissemination of OXA-24type beta-lactamases are generally limited to Europe and the United States (7).

The production of carbapenemases is one of the main mechanisms that enable Acinetobacter spp. to become resistant to the more active carbapenems such as imipenem and meropenem (8). The chromosomally located OXA-51 beta-lactamase gene in A. baumannii may be mutated to up-regulate or hyper-produce enzyme that may result in reduced susceptibility to some carbapenems. Genes coding for related enzymes such as OXA-23 are encoded on plasmids that are readily transferable within species (9), and in response to increasing carbapenem use, the OXA-carbapenemases are now seen to be emerging as an important mechanism of carbapenem resistance among Acinetobacter spp. (4). Currently, the four main phylogenetic subgroups of OXA carbapenemases that are commonly found in Acinetobacter spp. include OXA-23,-24,-51,58-like enzymes (9). But OXA-type enzymes are an ever expanding group and it is likely that more will be discovered as demonstrated by the fact that between 2000 and 2006, a further six novel enzymes with carbapenemase properties including OXA-143 were described in Acinetobacter spp. (10).

The aim of this study was to determine if any OXA-carbapenemase genes could be detected among clinical isolates of MDR Acinetobacter spp. identified from patients at a TCHJ and to determine the relatedness of the MDR isolates.

\section{METHODOLOGY}


After obtaining ethical approval and while operating within the context in which this approval was granted, a total of 82 consecutive, de-duplicated MDR Acinetobacter spp. isolated from clinical specimens at a TCHJ between May 2009 and April 2010, were collected for the purposes of this study. The majority of these isolates were obtained from the intensive care unit (34\%) and the surgical wards $(32 \%)$. Laboratory tests for manual identification and susceptibility testing were performed according to CLSI (2009), and isolates were deemed to be MDR if resistant to one or more agents in three or more antimicrobial classes (11).

Conventional multiplex PCR was performed on all isolates to detect OXA-23, -24, -51, and -58 carbapenemase genes using primers published by Woodford et al (5). Pulse field gel electrophoresis (PFGE) using AscI DNA restriction was performed on the Acinetobacter isolates which shared phenotypic and or genotypic similarities.

\section{RESULTS}

The Acinetobacter spp. isolates were resistant to multiple antibiotics including ampicillin cotrimoxazole, gentamicin, ceftazidime, and ceftriaxone. The table below illustrates that most isolates were only susceptible to minocycline and polymixin B.

Key: minocycline-MH; polymixin B-PB; meropenem-MEM 
Thoms-Rodriguez et al

Table: Susceptibility pattern of the Acinetobacter isolates

\begin{tabular}{|c|c|c|c|c|c|c|c|c|}
\hline \multirow{2}{*}{$\begin{array}{l}\text { Susceptibility } \\
\text { Pattern }\end{array}$} & \multirow{2}{*}{$\begin{array}{l}\text { Percentage } \\
\text { of isolates } \\
n=82\end{array}$} & \multirow{2}{*}{\multicolumn{2}{|c|}{$\begin{array}{l}\text { OXA carbapenemase } \\
\text { gene patterns detected }\end{array}$}} & \multicolumn{5}{|c|}{ PFGE type } \\
\hline & & & & 1 & 2 & 3 & 4 & $\begin{array}{l}\text { Unknow } \\
\text { n }\end{array}$ \\
\hline \multirow{3}{*}{$\begin{array}{l}\text { Susceptible to } \mathrm{MH} \\
\text { and PB ONLY }\end{array}$} & $39 \%$ & OXA 51 only & $22 / 32$ & - & 22 & - & - & - \\
\hline & & OXA 51 and 24 & $9 / 32$ & 9 & - & - & - & - \\
\hline & & OXA 51 and 23 & $1 / 32$ & - & - & 1 & - & - \\
\hline \multirow{2}{*}{$\begin{array}{l}\text { Susceptible to MH, } \\
\text { PB and MEM only }\end{array}$} & $24.4 \%$ & OXA 51 only & $19 / 20$ & & & 1 & 4 & 14 \\
\hline & & OXA 51 and 24 & $1 / 20$ & 1 & - & - & - & - \\
\hline Pandrug resistant & 1.2 & OXA 51 only & $1 / 1$ & - & - & 1 & - & - \\
\hline \multirow[t]{3}{*}{ Other } & 35.4 & OXA 51 only & $26 / 29$ & 2 & 0 & 9 & 3 & 12 \\
\hline & & OXA 51 and 24 & $2 / 29$ & - & - & 2 & - & - \\
\hline & & $\begin{array}{l}\text { OXA } \\
51 \text { and } 58\end{array}$ & $1 / 29$ & - & - & 1 & - & - \\
\hline
\end{tabular}

Table showing the percentage of Acinetobacter spp. isolates resistant to the panel of antibiotics tested. 54\% were resistant to meropenem. Eighty one isolates were positive for blaoxA-51, regardless of whether the isolate was susceptible or resistant to meropenem. In addition 12of these isolates also had blaOXA-24, one had blaoxA-23, and one had blaoxA-58. Of these isolates but two (blaoxA-24 and blaoxA-58) were resistant to meropenem. One isolate had a blaoxA-23 alone. The dendograms shown in the figure illustrate that there are at least three distinct clonally related groups of the Acinetobacter isolates present in the study population. 


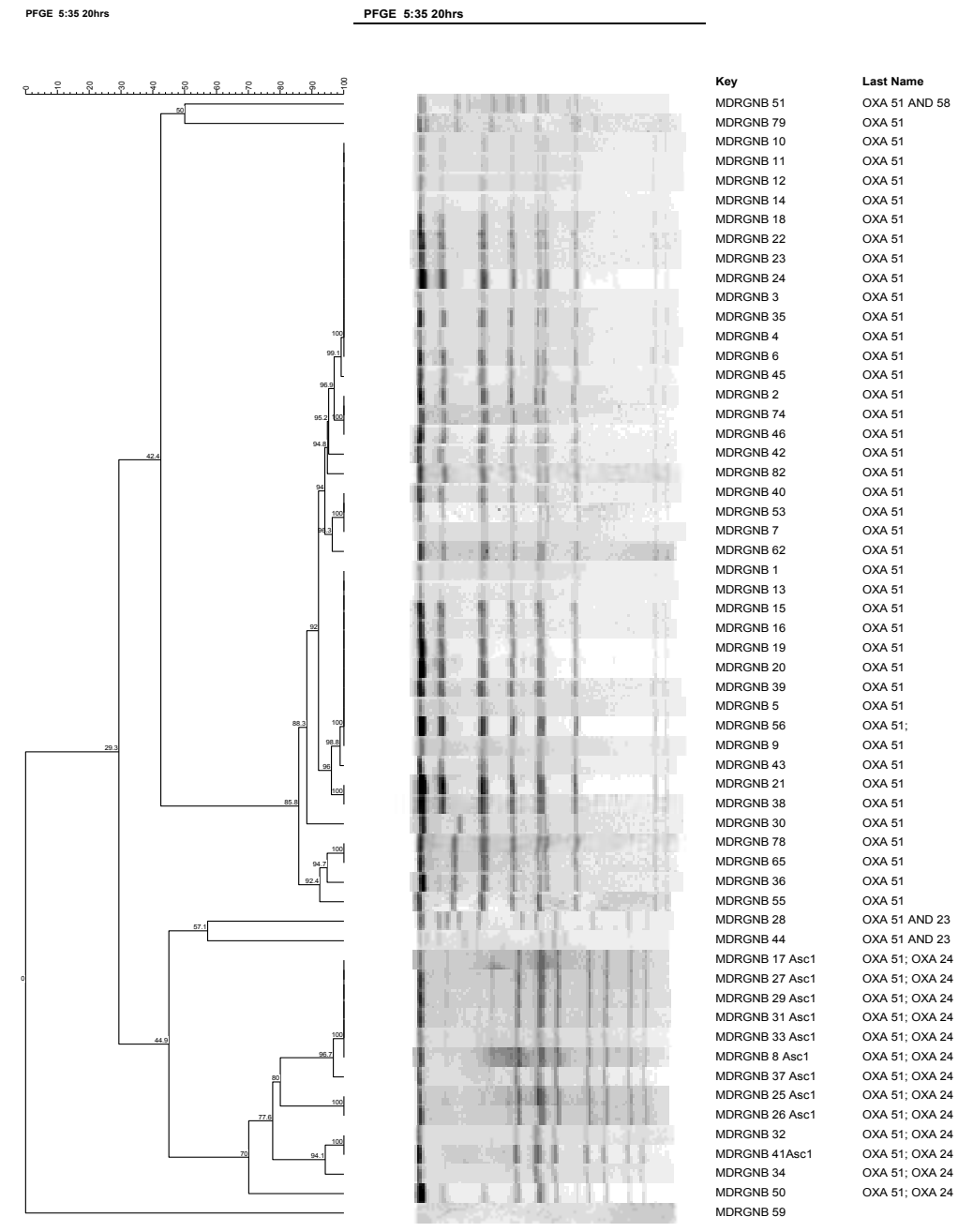

Figure: Acinetobacter PFGE results

PFGE of Acinetobacter baumannii identified two epidemic lineages, and some sporadic isolates at TCHJ. The most prevalent involved 40 isolates that shared $>85 \%$ similarity and were positive for only OXA-51, a gene common to all A. baumannii; this PCR profile was shared by only one other isolate unrelated by PFGE. The second epidemic cluster comprised 13 isolates, all of which tested positive for the OXA-24 carbapenemase gene in addition to OXA-51. The two $A$. baumannii positive for the OXA-23 carbapenemase and the single isolate positive for the OXA- 
58 carbapenemase, were unrelated to each other and to the two epidemic clusters. While the latter appear to be new introductions, the major clusters comprised isolates derived from multiple years, which indicates that they formed parts of ongoing outbreaks or it may be that these strains have already become endemic in the absence of adequate infection control.

\section{DISCUSSION}

Multiplex PCR identified OXA carbapenemase genes as a common mechanism of carbapenem resistance among isolates of Acinetobacter spp. at a TCHJ. Almost all (98.7\%) of these isolates had the blaoxa51 gene typically found in Acinetobacter baumannii species (5). The gene may be unique to this species and could be used as a means of rapidly identifying them (5). The presence of blaoxA51 does not automatically mean its phenotypic expression as it has to be regulated by insertion sequences such as ISAba1 (5). This was not evaluated and is one of the limitations of this study this study. A further limitation is that all the possible mechanisms for carbapenem was not explored. Although the impact of the regulation and over expression of the blaoxA51 gene has not been fully determined, recent data suggests that it is involved in carbapenem resistance (10). The high prevalence of carbapenem resistance seen among these Acinetobacter spp. could therefore be related to the presence and expression of blaOXA51 however further studies are needed to confirm this finding.

Reduced susceptibility or total resistance to carbapenems is usually seen when alleles encoding OXA-23 like, OXA-24 like and OXA-58 like genes are present in Acinetobacter spp. This was true for the 5 isolates that had these genes present.

PFGE revealed at least three groups of Acinetobacter spp. that were highly related which suggests that they either were derived from an outbreak or were endemic strains (12). 
Longitudinal epidemiological studies are needed to determine this. Among the clonally related isolates, $38 \%$ were from the intensive care unit and $28 \%$ came from surgical wards. The rest were evenly distributed throughout the rest of the hospital.

\section{CONCLUSION}

The increased resistance to carbapenem antibiotics remains a cause for concern as this class of antibiotics remains one of the few therapeutic options for the treatment of infections caused by MDR resistant organisms (1). As resistance to these antibiotics increases, therapeutic options become more limited. Isolates that become endemic in a healthcare setting pose a risk of infection patients who receive care at that institution resulting in increased morbidity and mortality (4). An understanding of the prevalence and mechanism of carbapenem resistance in a local setting is important to drive antibiotic policies to limit the emergence of further resistance while ensuring adequate therapy (1). As many of the genes coding for some of the OXA carbapenemases are present on mobile genetic elements, infection control will play a key role in limiting the spread of some of these resistance genes. This is particularly important in the context of decreased antimicrobial therapeutic options $(1,5)$.

\section{ACKNOWLEDGEMENTS}

This project was supported by the Scotiabank Jamaica Foundation. This institution only provided financial support and played no part in the study design, its execution or publication. We thank John Lindo, Department of Microbiology, University of West Indies, for editorial contributions. 


\section{AUTHORS' NOTE}

There are no financial or personal relationships with other persons or organizations that would inappropriately influence this study. 


\section{REFERENCES}

1. Hawkey PM. The growing burden of antimicrobial resistance. J Antimicrob Chemother 2008; 62: i1-i9.

2. Nicholson AM, Ledgister S, Williams T, Robinson S, Gayle P, Lindo T et al. Distribution of nosocomial organisms and their resistance patterns in The Intensive Care Unit of the University Hospital of the West Indies, Kingston, Jamaica. West Indian Med J 2009; 58: 0043-3144.

3. Mendes RE, Spanu T, Deshpande L, Castanheira M, Jones RN, and Fadda G. Clonal dissemination of two clusters of Acinetobacter baumannii producing OXA-23 or OXA-58 in Rome, Italy. Clin Microbiol Infect 2009; 15: 588-92.

4. Brown S, and Sebastian A. OXA beta-lactamases in Acinetobacter; the story so far. J Antimicrob Chemother 2006; 57: 1-6.

5. Woodford N, Ellington MJ, Coelho JM, Turton JF, Ward ME, Brown S, et al. Multiplex PCR for genes encoding prevalent OXA carbapenemases in Acinetobacter spp. Int $\mathrm{J}$ Antimicrob Agents 2006; 27: 351-3

6. Wang H, Guo P, Sun H, Wang H, Yang Q, Chen M, Xu, et al. Molecular epidemiology of clinical isolates of carbapenem-resistant Acinetobacter spp. from Chinese hospitals. Antimicrob Agents Chemother 2007; 51: 4022-8.

7. Manchanda V, Sanchaita S, and Singh NP. Multidrug Resistant Acinetobacter. J Glob Infect Dis 2010; 2: 291-304.

8. Barker Keith F. Antibiotic resistance: a current perspective. Br J Clin Pharmacol 1999; 48: $109-24$. 
9. Queenan AM, Bush K. Carbapenemases: the versatile beta-lactamases Clin Microbiol Rev 2007; 20: 440-58.

10. Higgins PG, Poirel L, Lehmann M, Nordmann P, Seifert H. OXA-143, a Novel Carbapenem-Hydrolyzing Class D $\beta$-Lactamase in Acinetobacter baumannii. Antimicrob Agents Chemother 2009; 53: 5035-8.

11. Souli M, Galani I, and Giamarellou H. Emergence of extensively drug-resistant and pan drug-resistant Gram-negative bacilli in Europe. Euro Surveill 2008; 1-11.

12. Tenover FC, Arbeit RD, Goering R V, Mickelsen PA, Murray BE, Persing DH, et al. Interpreting patterns produced by pulsed-field gel electrophoresis: criteria for bacterial strain typing. J Clin Microbiol 1995; 33: 2233-9. 